\title{
Malaria in Pregnancy: an overview
}

\author{
Alex C. Samak*
}

\section{INTRODUCTION}

Malaria is a parasitic disease affecting red blood cells that is transmitted by female mosquitoes of the Anopheles genus. A. gambiae and A. funestus are the main vectors of the disease in Africa (1). Following infection by sporozoites found in the mosquito's salivary glands, the host's liver cells are initially used by the parasite to grow and multiply. Parasites released into the circulation subsequently infect red blood cells, proliferate in them and eventually cause them to burst. Of the four parasitic protozoa causing malaria, Plasmodium falciparum, $P$. vivax, $P$. malariae, and $P$. ovale, $P$. falciparum is the most common and the most dangerous, causing between 700,000 and 2.7 million deaths annually, most of which are in children and pregnant mothers (1). The millions of pregnant women residing in regions where malaria is endemic are particularly vulnerable to the effects of $P$. falciparum infection. The severity of malarial infections is also considerably increased in pregnant women and their unborn children as a specific form of the disease afflicts them: placental malaria. This review aims at introducing the reader to the implications of malaria infection during pregnancy.

\section{THE PLACENTA AND IMMUNE EVASION}

Pregnancy causes a number of physiological changes that affect the way the Plasmodium parasite invades its host. Down regulation of normal maternal immune response is necessary to prevent rejection of the conceptus. Cell-mediated immunity (Th1) is particularly suppressed during pregnancy, and the mother is increasingly reliant on humoral immunity (Th2) for protection. It was believed that this suppression accounts for pregnant women's increased risk of malaria infection. However, it has been shown

\footnotetext{
* To whom correspondence should be addressed: Alex C. Samak, 7667, Saint-Denis, Montréal, Québec, H2R 2E7 email: alexandre.cengarle-samak@mail.mcgill.ca
}

that despite this depression, the maternal immune system continues to respond to the parasite and that antibodies preventing $P$. falciparum's attachment to the placenta can be produced and correlate with better outcomes for the fetus (2). Moreover, significant variations in risk and severity of infection between women in their first pregnancy (primigravides) and women having had multiple pregnancies (multigravides) have been reported, with risk and severity decreasing in proportion to the number of pregnancies $(3,4)$. These observations are not explained by mothers' suppressed immune system, but suggest that immune build-up is achieved after several pregnancies and infections.

In placental malaria, it has been suggested that the parasite can temporarily evade the immune system by hiding within the vascular placenta found only in pregnant women (5). Indeed, while peripheral circulation can be free of parasites, high placental parasitation is sometimes observed in infected mothers (6). Although both $P$. vivax and $P$. falciparum can be found in the placenta, $P$. falciparum is sequestered there more frequently and at higher density (7). Red blood cells infected by $P$. falciparum are sequestered in the placenta by binding to membrane proteins expressed specifically on its endothelium $(8,9)$. P. falciparum is known to express variant proteins on the surface of infected erythrocytes (IEs). These proteins can interact with various endothelial proteins, resulting in the adherence of IEs to endothelium and their sequestration in organs. Chondroitin sulphate A (CSA)(8) and hyaluronic acid (HA)(9) are proteins expressed by placental endothelium to which proteins expressed by IEs can bind. The sub-population of IEs capable of biding will be preferentially sequestered in the placenta and thereby temporarily evade an immune response. Because the sub-population of parasites causing IEs binding to CSA should not usually infect non-pregnant hosts (8), primigravides have never been immunologicaly 
primed against this particular sub-population.

Eventually, the mother develops an effective humoral response to placental malaria by producing antibodies inhibiting IEs binding to placental proteins such as CSA (10). In a study recently conducted in western Kenya, both infected and non-infected women showed increased production of these antibodies, which was associated with increased birth weight and gestational age of newborns (11). Indeed, the study showed that secundigravides producing anti-adhesion antibodies delivered babies were on average, $398 \mathrm{~g}$ heavier and 2 weeks more mature than babies delivered to secundigravides without anti-adhesion activity.

Why the placenta offers a favorable milieu for immune evasion is still debated. Some contend the placenta is immunologically malaria-naïve (12) while others suggest that immunosuppressive factors synthesized locally - estrogens, for example - act with more vigor locally, thereby allowing the parasite to thrive (13). According to the former explanation, longer exposure periods are necessary for the maternal immune system to recognize placenta-sheltered parasites and respond appropriately. According to the latter, reduction of cortisol and estrogen levels with higher parity reduces vulnerability. This question is likely to remain unresolved until the exact mechanisms providing immune privilege to the fetus by its immediate environment are elucidated.

Unfortunately, placental malaria is alarmingly common in endemic regions of sub-Saharan Africa, as was shown by a study conducted in Tanzania showing evidence of malaria infection in $75.5 \%$ of placenta samples (5). In Kenya, active or past malaria was detected in 64\% primigravides (14). Placental malaria prevalence can, however, vary greatly from region to region. A French team working in Madagascar found prevalence to vary from $2.1 \%$ in the eastern unstabletransmission region to $26.2 \%$ in the western part of the island (15). Nevertheless, infection, in any region, carries important risks.

\section{PLACENTAL MALARIA AND POOR PREGNANCY OUTCOMES}

Pregnancy malaria is a direct cause of numerous poor outcomes for both mother and fetus. Maternal effects include anemia, cerebral malaria, pulmonary edema and kidney failure $(3,4,14)$. Poor outcomes for the fetus include abortion, stillbirth, premature delivery, lowbirth weight (LBW), and intrauterine growth retardation $(4,5,14,16,17)$.

The Plasmodium parasite's life cycle requires it to multiply within IEs, and ultimately cause them to burst. $P$. falciparum is known to be able to infect up to $5 \%$ of a host's red blood cells. The direct destruction of such an important number of IEs is an important cause of malarial anemia. However, it is not the only one. A recent study showed $P$. falciparum produced modifications in both IEs and uninfected erythrocyte (UEs) membranes (18). These changes, which mimic red blood cell's normal aging process, are attributed to oxidative-stressed induced by $P$. falciparum infection. UEs exposed to IEs become old prematurely and are cleared by the host's reticulo-endothelial system, thus contributing to malarial anemia. 75,000 to 200,000 infant deaths are estimated to be related to pregnancy malaria in Sub-Saharan Africa (19).

Anemia (hemoglobin $[\mathrm{Hg}]<11 \mathrm{~g} / \mathrm{dL}$ ) is associated with active pregnancy malaria. This association is similar in primigravides and multigravides (14). The adjusted odds ratio $(\mathrm{OR})$ for severe anemia $([\mathrm{Hg}]<7 \mathrm{~g} / \mathrm{dL})$ in gravidities 1 to 4 during active infection is 2.21 (CI: $1.36-3.61$ ) (14). Similar results were found in a study in Gabon, where infection was associated with anemia in all gravidities with moderately $([\mathrm{Hg}]<7-9 \mathrm{~g} / \mathrm{dL})$ or severely anemic mothers having higher parasite densities than women with low anemia (20). Severe maternal anemia in turn has major and harmful effects on the fetus' development, and has been linked, independently from its etiology, to LBW, prolonged labor, increased induction rates and operative deliveries (21).

As stated earlier, during healthy pregnancies, Th1 immune actors are subdued, and $\mathrm{Th} 2$ actors more relied upon. Th1 cytokines are embryotoxic and in rodent models, they are known to induce spontaneous abortions or still-births (22). It has been shown that placental malaria leads to an increase of Th1 cytokines in the placenta $(23,24,25)$. This cytokine-imbalance has been linked to intra-uterine growth retardation and premature birth (12). A study comparing placental cytokines from women living in nonmalarious and malaria-holoendemic regions found Th1 cytokines to be absent in the former group, and abundant in the latter (23). IFN- $\gamma$ and IL-2 were only present in women exposed to malaria. These pro-inflammatory factors and other immune responses to malaria infection are thought to be associated with syncytiotrophoblast necrosis, irregular thickening and scarring of trophoblastic membrane, and breakdown of placental integrity observed in parasitized placentas $(16,26)$. This in turn impairs maternal-fetal nutrient and metabolite exchanges, and impedes normal growth. Placental malaria also induces production of chemokines recruiting and activating monocytes(25). Monocyte infiltration and accumulation as macrophages in intervillous space is another risk factor for low birth weight. Indeed, although macrophages play an important role in controlling the parasitic infection, a number of inflammatory mediators they produce can also damage tissues.

Thus, while the safe haven afforded to the parasite by 
the placenta probably increases the severity of the mother's disease, the Th1 immune response taking place in the placenta accounts for poor pregnancy outcomes. Newborns having active placental malaria were found to have a significantly lower weight than those having had past placental malaria or no infection (27). LBW has been repeatedly and consistently associated with placental malaria $(4,5,14,17)$. In regions of stabletransmission malaria (high exposure, adult women more likely to have acquired immunity), primigravides were more likely to have LBW babies than multigravides $(5,14,17)$. On the other hand, multigravides were not found to be protected in regions of unstabletransmission (low exposure adult women less likely to have acquired immunity), presumably because the level of exposure required for multigravides to gain immune competence is only reached in stable-transmission regions (17). Long-term effects of placental malaria have been reported: anemia and malaria infection are more common at 2 and 6 months of age respectively, if the child was born to a mother with placental malaria(28). Maternal infection within one week of delivery was linked to increased risk of infant death (OR: 4, CI: 1.2 - 13.7) (29).

P. falciparum, malariae and ovale parasites can be found in umbilical-cord blood of infected mothers, which could be transmitted directly to the fetus. Although there are conflicting results on the possibility of congenital malaria $(30)$, there are cases $(31,32)$ and a survey in sub-Saharan Africa (33) that report newborns being congenitally infected. Unfortunately, more research is needed in order to guide health policy and to develop treatments for congenital transmission.

\section{WORKING AGAINST A VICIOUS CIRCLE}

There seems to exist an alarming synergism between infectious diseases working against health-care workers' efforts to immunize vulnerable populations. Of the 5 million neonates estimated to die each year, $30-40 \%$ succumb to infectious diseases (34). Neonates are greatly dependant of maternal immunoglobulins transferred during gestation for protection against infectious agents. The mother passes to her child antibodies that her immune system produces in response to infection or supervised immunization (i.e.: vaccination). Immunization of pregnant women living in developing countries is thus crucial to diminish the number of neonates dying of infectious diseases.

It is now recognized that immunization programs in malaria-endemic regions will have to take into account the adverse effects of malaria infection. First, malaria impedes materno-fetal antibody transfer, thereby decreasing the effectiveness of the natural immunological development of the fetus. Placental malaria infection was found to reduce materno-fetal antibody transfer for herpes simplex virus 1 by $69 \%$ (35). This reduction has been attributed to the premature birth caused by placental malaria, which is known to result in decreased materno-fetal antibody transfer (36), and also to the direct competitive-inhibition of immunoglobulin selective transport to fetus by elevation of malaria specific and non-specific immunoglobulins caused by infection (37). By the same token, placental malaria decreases materno-fetal transfer of antibodies developed by the mother following vaccine-immunization. This likely will affect the efficacy of immunization campaigns targeting neonates via pregnant women. It is also reasonable to expect that malarial infection inhibits immune response to vaccination directly in the mother. Indeed, studies have shown that adults and children on anti-malarial chemoprophylaxis developed enhanced antibody immunity compared to non-treated subjects, in response to tetanus toxoid and diphtheria, and tetanus toxoid and meningococcus vaccination, respectively (37). Finally, the newborn's immune system has been shown to be adversely affected by malaria infection during pregnancy (38). IFN-production by T-lymphocytes was decreased, possibly resulting in an impaired Th1 differentiation and weakened cell-mediated immune response to infection. This puts newborns, who are already vulnerable, at further risk of infection.

\section{DISEASE-DISEASE INTERACTIONS: MALARIA AND HIV}

Synergism between malaria and HIV infection is yet another dreaded possibility. HIV infects, disables and ultimately destroys CD4+ lymphocytes essential for the development of anti-malaria antibodies. It has thus been hypothesized HIV infection could increase the risk of concomitant malaria infection, parasitemia, morbidity and mortality.

Indeed, Steketee et al. report HIVSP multigravides had a significantly higher parasitemia prevalence than HIVSN multigravides $(52.9 \%$ to $28.5 \%$ OR : 1.85 , CI : 1.46-2.35) and higher incidence of placental malaria in HIVSP multigravides than HIVSN multigravides (34.8\% to $12.4 \%$ OR:1.85, CI : 1.46-2.35) (39). Recent studies corroborate this finding. Antibodies against variant surface antigens (VSA) on IEs (binding CSA) thought to protect against placental malaria were found to be lower in pregnant HIVSPs than in pregnant HIVSNs, and inversely proportional to viral load (40). Decreased materno-fetal transfer of antibodies to certain malaria antigens were observed in HIVSPs compared to HIVSNs (41).

The hypothesis that malaria infection in pregnancy can facilitate mother-to-child transmission (MTCT) of 
HIV has also been explored, but remains controversial. A randomized control trial at Johns Hopkins University found the risk of MTCT associated with placental malaria was 2.89 (42) while the International Centre for Reproductive Health noted no correlation between placental malaria and in utero or peripartal transmission of HIV-1 (43).

The results presented above indicate HIV infection puts pregnant women at increased risk of developing malaria. HIV prevalence in pregnant women reaches as high as $25-45 \%$ in sub-Saharan Africa $(39,41)$, a part of the continent comprising numerous high malaria endemic zones. On the other hand, malaria infection could increase HIV MTCT, further aggravating the AIDS epidemic. The measures to approach the complex interplay between the two diseases are not readily apparent. Total eradication of both is of course the ultimate goal. As such, any studied endeavor to reduce their transmission is desirable. However, the importance of the impact of reducing HIV prevalence in pregnant women on placental malaria remains unclear. Studies comparing malaria infection in HIVSP gravides on triple therapy and in HIVSP gravides not on therapy could yield crucial information and help direct future public health policy. Even if HIV significantly affected malaria prevalence, measures to reduce it should include vector-control and the development of a vaccine.

\section{WHERE WE STAND: PREVENTION, VACCINES, POLITICAL ACTION}

In the face of mounting evidence of the relative failure of traditional chloroquine malaria chemoprophylaxis in pregnant women $(20,42)$ and of logistics/financial reasons, the World Health Organization (WHO) has put forward new guidelines for combating and preventing malaria during pregnancy (45). These recommend that women living in high transmission areas in Africa receive intermittent preventive treatment (IPT) with an effective antimalarial agent such as sulfadoxine-pyrimethamine (SP) at scheduled antenatal visits. All pregnant women in targeted areas should undergo at least two sessions of IPT after first fetal movements. SP ITP is associated with a reduction in placental malaria in HIVSNs, but not in HIVSPs (46). SP should not be administered more than once a month, and not be given during the first trimester, since it is an anti-folate drug and so a potential risk for congenital defects. Preventive measures that can be used during the first trimester include chloroquine, quinine, quinide and insecticidetreated nets $(46,48)$.

Unfortunately, implementation of the WHO guidelines is burdened by the notorious problems complicating health service delivery in the developing world: the logistical challenges of reaching remote regions, resource scarcity, lack of infrastructure, etc. However, the size of the population affected - more than $40 \%$ of all births worldwide occur in areas with endemic malaria (48)-malaria's well documented effects on both mother and fetus, and the apparent synergistic interplay between it and various infections make it critical to push for full implementation. ITP and some forms of chemoprophylaxis have been demonstrated to have a dramatic positive impact on the outcome of newborns, such as LBW (49).

Parallel efforts to control pregnancy malaria are focused on the development of effective vaccines. As was stated above, placental malaria arises when infected red blood cells segregate in the placenta by binding the CSA and HA receptors $(8,9)$. Malaria resistance in multigravides has been linked to the presence of antibodies targeting binding-proteins found on the surface of IEs that permit placenta-segregation $(10,11)$. These antibodies prevent adhesion and clustering of IEs in the placenta, protecting the mother and the child from severe morbidity associated with placental malaria. $P$. falciparum erythrocyte membrane protein 1 (PfEMP1), a subdomain of which binds CSA (50), is being investigated as a candidate for a placental malaria vaccine. Eliciting production of anti-adhesion antibodies by vaccination would provide immune-naïve women the protection resistant multigravides benefit from. A pregnancy malaria vaccine could save thousands of lives every year and boost the efficiency of immunization campaigns in malaria-endemic regions. Such a vaccine, however, would only be useful in preventing placental malaria. Although current research is focusing on PfEMP1, multiple parasite-encoded antigens known as variant surface antigens (VSA) are potential targets, as they cause IEs segregation in other organs. The isolation of a VSA expressed by most or all sub-population IEs and not by normal human cells would be the first step in the development of a truly "broad-spectrum" malaria vaccine.

The optimism of these scientific advances should not cloud the socio-economical importance to public health. Without resources, it is impossible for any government to provide its citizens with adequate care, especially when facing robust and vicious diseases like malaria and HIV. As was recognized by the World Trade Organization's (WTO) General Assembly in August 2003, the developed world's governments should allow and facilitate the transfer of some of these resources to needy nations at a minimal cost.

Canada just recently became the first country to implement the WTO's decision by passing Bill C-9 An Act to amend the Patent Act and the Food and Drugs 
Act (which received Royal Assent on May 14th 2004). This bill allows Canadian pharmaceutical companies to seek compulsory licenses to produce patented medicines and export them to eligible countries. The bill includes a list of 56 covered medicines (Schedule 1) and gives the federal Cabinet the authority to add products to this list. Schedule 1 is based on the World Health Organisation's Model List of Essential Medicines. Although none of the products included on the list can be matched to antimalarials catalogued by Winstanley et al. in their review on the subject $(51,52)$, some antibiotics that are included can be put to use in combination with antimalarials for better treatment (52). It is important to note that production of most antimalarials is today free of patent restrictions.

However, newly developed drugs-for example designed to fight placental malaria-will not automatically be available for compulsory licensing. Indeed, recommendations by the ministers of health and industry are initially required for the federal Cabinet to add a product to Schedule 1. Such an addition could face the opposition of interested pharmaceutical companies and ultimately be defeated. This was the fate of the motion introduced by the New Democratic Party to add to Schedule 1 medicines used to treat community-acquired pneumonia (53). Many share the fear that the pharmaceutical lobby will end up neutralizing the present form of Bill C-9. For this reason, NGOs and other civil society organizations are pressuring the government to abolish Schedule 1 and to modify a number of other controversial aspects of the bill.

\section{CONCLUSION}

Malaria infection during pregnancy has important adverse effects on both mother and fetus, and causes a great number of deaths around the world. Fortunately, many of these deaths could be avoided each year by prophylaxis programs and parallel preventive measures. Research and public health policy should be geared towards prevention of malaria infection during pregnancy.

\section{REFERENCES}

1. National Center for Infectious Diseases, Division of Parasitic Diseases. Anopheles Mosquitoes. April 23, 2004. http://www.cdc.gov/malaria/biology/mosquito/

2. Duffy P, Fried M. Antibodies That Inhibit Plasmodium falciparum Adhesion to Chondroitin Sulfate A Are Associated with Increased Birth Weight and the Gestational Age of Newborns. Infec. and Immu. 71(11): 6620-3; 2003

3. Shulman CE, Graham WJ, Jilo H, et al. Malaria is an important cause of anaemia in primigravidae: evidence from a district hospital in coastal Kenya. Trans R Soc Trop Med Hyg. 90(5):535-9; 1996

4. Steketee RW, Wirima JJ, Hightower AW, et al. The effect of malaria and malaria prevention in pregnancy on offspring birthweight, prematurity, and intrauterine growth retardation in rural Malawi. Am J Trop Med Hyg. 55(1 Suppl):33-41; 1996

5. Menendez C, Ordi J, Ismail MR, et al. The impact of placental malaria on gestational age and birth weight. J Infect Dis. 181(5):1740-5; 2000.

6. Ismail MR, Ordi J, Menendez C, et al. Placental pathology in malaria: an histological, immunohistochemical and quantitative study. Human Pathology 31(1):85-93; 2000

7. McGready R, Davison BB, Stepniewska K, et al. The effects of Plasmodium falciparum and $\mathrm{P}$. vivax infections on placental histopathology in an area of low malaria transmission. Am J Trop Med Hyg. 70(4):398-407; 2004

8. Fried M, Duffy PE. Adherence of Plasmodium falciparum to chondroitin sulfate $\mathrm{A}$ in the human placenta. Science 272(5267):1502-4; 1996

9. Beeson JG, Rogerson SJ, Cooke BM, et al.. Adhesion of Plasmodium falciparum-infected erythrocytes to hyaluronic acid in placental malaria. Nat Med. 6(1):86-90; 2000

10. Fried M, Nosten F, Brockman A, et al. Maternal antibodies block malaria. Nature 395(6705):851-2; 1998

11. Duffy PE, Fried M. Antibodies that inhibit Plasmodium falciparum adhesion to chondroitin sulfate $\mathrm{A}$ are associated with increased birth weight and the gestational age of newborns. Infect Immun. 71(11):6620-3; 2003

12. McGregor, I.A. Epidemiology, malaria and pregnancy. Am. J. Trop. Med. Hyg. 33 (4), 517-525; 1984

13. Rasheed, F.N., Bulmer, J.N., Dun, D.T.,et al. Suppressed peripheral and placental blood lymphoproliferative responses in first pregnancies: relevance to malaria. Am. J. Trop. Med. Hyg. 48(2):154-60; 1993

14. Shulman, C. E. , Marshall, T. , Dorman, E. K., et al. Malaria in pregnancy: adverse effects on haemoglobin levels and birthweight in primigravidae and multigravidae. Tropical Medicine \& International Health 6 (10): 770-778; 2001

15. Cot M, Brutus L, Pinell V, et al.. Malaria prevention during pregnancy in unstable transmission areas: the highlands of Madagascar. Trop Med Int Health. 7(7):565-72; 2002

16. Okoko BJ, Enwere G, Ota MO. The epidemiology and consequences of maternal malaria: a review of immunological basis. Acta Trop. 87(2):193-205; 2003

17. Newman RD, Hailemariam A, Jimma D, et al. Burden of malaria during pregnancy in areas of stable and unstable transmission in Ethiopia during a nonepidemic year. J Infect Dis. 187(11):1765-72; 2003

18. Omodeo-Sale F, Motti A, Basilico N, et al. Accelerated senescence of human erythrocytes cultured with Plasmodium falciparum. Blood 102(2):705-11; 2003

19. Steketee RW, Nahlen BL, Parise ME, et al. The burden of malaria in pregnancy in malaria-endemic areas. Am J Trop Med Hyg. 64(1-2 Suppl):28-35; 2001

20. Bouyou-Akotet MK, Ionete-Collard DE, Mabika-Manfoumbi $\mathrm{M}$, et al. Prevalence of Plasmodium falciparum infection in pregnant women in Gabon. Malar J. 2(1):18; 2003

21. Malhotra M, Sharma JB, Batra S, et al. Maternal and perinatal outcome in varying degrees of anemia. Int J Gynaecol Obstet. 79(2):93-100; 2002

22. Vassiliadis, S., D. Tsoukatos, I. Athanassakis. Interferoninduced class II expression at the spongiotrophoblastic zone of the murine placenta is linked to fetal rejection and developmental abnormalities. Acta Physiol Scand. 151(4):485; 1994

23. Fried M, Muga R, Misore A, et al. Malaria Elicits Type 1 Cytokines in the Human Placenta:IFN-g and TNF-a Associated with Pregnancy Outcomes. Jour. of Immunol. 160(5): 2523-30; 1998 
24. Suguitan AL Jr, Leke RG, Fouda G, et al. Changes in the levels of chemokines and cytokines in the placentas of women with Plasmodium falciparum malaria. J Infect Dis. 188(7):1074-82; 2003

25. Abrams ET, Brown H, Chensue SW, et al. Host response to malaria during pregnancy: placental monocyte recruitment is associated with elevated beta chemokine expression. J Immunol. 170(5):2759-64; 2003

26. Crocker IP, Tanner OM, Myers JE, et al. Syncytiotrophoblast degradation and the pathophysiology of the malaria-infected placenta. Placenta 25(4):273-82; 2004

27. Leopardi O, Naughten W, Salvia L, et al. Malaric placentas. A quantitative study and clinico-pathological correlations. Pathol Res Pract. 192(9):892-8; 1996

28. LeHesran JY, Cot M, Personne P, et al. Maternal placental infection with Plasmodium falciparum and malaria morbidity during the first 2 years of life. Am J Epidemiol 146(10):826-31; 1997

29. Luxemburger C, McGready R, Kham A, et al. Effects of malaria during pregnancy on infant mortality in an area of low malaria transmission. Am J Epidemiol. 154(5):459-65; 2001

30. Tobian AA, Mehlotra RK, Malhotra I, et al. Frequent umbilical cord-blood and maternal-blood infections with Plasmodium falciparum, P. malariae, and P. ovale in Kenya. J Infect Dis. 182(2):558-63; 2000

31. Hewson M, Simmer K, Blackmore T. Congenital malaria in a preterm infant. J Paediatr Child Health. 39(9):713-715; 2003

32. Olowu WA, Torimiro SE. Congenital malaria in 8 hours old newborn: case report. Niger J Med. Apr-11(2):81-2; 2002

33. Fischer PR. Congenital malaria: an African survey. Clin Pediatr (Phila). 36(7):411-3; 1997

34. Stoll BJ. The global impact of neonatal infection. Clin Perinatol 1997;24(1):1-21. In: Duffy P. Maternal immunization and malaria in pregnancy. Vaccine. 2003; 21: 3358-3361.

35. Okoko BJ, Wesumperuma LH, Ota MO, et al. The influence of placental malaria infection and maternal hypergammaglobulinemia on transplacental transfer of antibodies and $\operatorname{IgG}$ subclasses in a rural West African population. J Infect Dis. 184(5):627-32; 2001

36. Okoko BJ, Wesumperuma LH, Hart AC. Materno-foetal transfer of $\mathrm{H}$. influenzae and pneumococcal antibodies is influenced by prematurity and low birth weight: implications for conjugate vaccine trials. Vaccine 20(5-6):647-50; 2001

37. Duffy P. Maternal immunization and malaria in pregnancy. Vaccine. 2003; 21(24): 3358-61.

38. Ismaili J, van der Sande M, Holland MJ, et al. Plasmodium falciparum infection of the placenta affects newborn immune responses. Clin Exp Immunol. 133(3):414-21; 2003

39. Steketee RW, Wirima JJ, Bloland PB, et al. Impairment of a pregnant woman's acquired ability to limit Plasmodium falciparum by infection with human immunodeficiency virus type-1. Am J Trop Med Hyg 55(1 Suppl):42-9; 1996

40. Mount AM, Mwapasa V, Elliott SR, et al. Impairment of humoral immunity to Plasmodium falciparum malaria in pregnancy by HIV infection. Lancet 363(9424): 1860-7; 2004

41. Ayisi JG, Branch OH, Rafi-Janajreh A, et al. Does infection with Human Immunodeficiency Virus affect the antibody responses to Plasmodium falciparum antigenic determinants in asymptomatic pregnant women? J Infect. 46(3):164-72; 2003

42. Brahmbhatt H, Kigozi G, Wabwire-Mangen F, et al. The effects of placental malaria on mother-to-child HIV transmission in Rakai, Uganda. AIDS 17(17):2539-41; 2003

43. Inion I, Mwanyumba F, Gaillard P, et al. Placental malaria and perinatal transmission of human immunodeficiency virus type 1 . J Infect Dis. 188(11):1675-8; 2003

44. Sirima SB, Sawadogo R, Moran AC, et al. Failure of a chloroquine chemoprophylaxis program to adequately prevent malaria during pregnancy in Koupela District, Burkina Faso. Clin Infect Dis. 36(11):1374-82; 2003

45. Strategic framework for malaria control during pregnancy in the WHO African Region. Geneva: World Health Organization; 2002.

46. van Eijk AM, Ayisi JG, ter Kuile FO, et al. Effectiveness of intermittent preventive treatment with sulphadoxinepyrimethamine for control of malaria in pregnancy in western Kenya: a hospital-based study. Trop Med Int Health 9(3):35160; 2004

47. Newman RD, Parise ME, Slutsker L, et al. Safety, efficacy and determinants of effectiveness of antimalarial drugs during pregnancy: implications for prevention programmes in Plasmodium falciparum-endemic sub-Saharan Africa. Trop Med Int Health. 8(6):488-506; 2003

48. Stoll BJ. The global impact of neonatal infection. Clin Perinatol. 24(1):1-21; 1997

49. Steketee RW, Wirima JJ, Hightower AW, et al. The effect of malaria and malaria prevention in pregnancy on offspring birthweight, prematurity, and intrauterine growth retardation in rural Malawi. Am J Trop Med Hyg. 55(1 Suppl):33-41; 1996

50. Buffet PA, Gamain B, Scheidig C, et al. Plasmodium falciparum domain mediating adhesion to chondroitin sulfate A: a receptor for human placental infection. Proc Natl Acad Sci USA 96(22):12743-8; 1999

51. Bill C-9 House of Commons. Canada.

52. Winstanley P, Ward S, Snow R, et al Therapy of falciparum malaria in sub-saharan Africa: from molecule to policy. Clin Microbiol Rev. 17(3):612-37; 2004

53. Global Access to Treatment: Canada's Bill C-9 and the Compulsory Licensing of Pharmaceuticals for Export to Countries in Need. Canadian HIV-AIDS Legal Network. July 2004.

Alex C. Samak is a second-year medical student at McGill University. He is interested in tropical and social medicine. He chairs on IFMSA-Quebec's Refugees and Peace Committee. 